\title{
Article \\ Collaborative Studies for the Detection of Taenia spp. Infections in Humans within CYSTINET, the European Network on Taeniosis/Cysticercosis
}

\author{
María Ángeles Gómez-Morales ${ }^{1, *}$, Patrizio Pezzotti ${ }^{1} \mathbb{D}$, Alessandra Ludovisi ${ }^{1}$, Belgees Boufana ${ }^{1,2} \mathbb{D}$, \\ Pierre Dorny ${ }^{3}$, Titia Kortbeek ${ }^{4}$, Joachim Blocher ${ }^{5}{ }^{\circledR}$, Veronika Schmidt ${ }^{6}{ }^{\circledR}$, Marco Amati ${ }^{1}$, Sarah Gabriël ${ }^{7}{ }^{(D}$, \\ Edoardo Pozio ${ }^{1,+}{ }^{+}$Andrea Sylvia Winkler $6,8,+$ and The Ring Trial Participants $\ddagger$
}

check for updates

Citation: Gómez-Morales, M.Á.; Pezzotti, P.; Ludovisi, A.; Boufana, B.; Dorny, P.; Kortbeek, T.; Blocher, J.; Schmidt, V.; Amati, M.; Gabriël, S.; et al. Collaborative Studies for the Detection of Taenia spp. Infections in Humans within CYSTINET, the

European Network on

Taeniosis/Cysticercosis.

Microorganisms 2021, 9, 1173.

https://doi.org/10.3390/

microorganisms 9061173

Academic Editor: Ronan Jambou

Received: 27 April 2021

Accepted: 26 May 2021

Published: 29 May 2021

Publisher's Note: MDPI stays neutral with regard to jurisdictional claims in published maps and institutional affiliations.

Copyright: (c) 2021 by the authors. Licensee MDPI, Basel, Switzerland. This article is an open access article distributed under the terms and conditions of the Creative Commons Attribution (CC BY) license (https:// creativecommons.org/licenses/by/ $4.0 /)$.
1 Department of Infectious Diseases, Istituto Superiore di Sanità, Viale Regina Elena 299, 00161 Rome, Italy; patrizio.pezzotti@iss.it (P.P.); alessandra.ludovisi@iss.it (A.L.); belgees.boufana@iss.it or belgees.boufana@apha.gov.uk (B.B.); marco.amati@iss.it (M.A.); edoardo.pozio@iss.it (E.P.)

2 National Reference Laboratory for Trichinella \& Echinococcus National Wildlife Management Centre (NWMC) Animal and Plant Health Agency Sand Hutton, York YO41 1LZ, UK

3 Veterinary Helminthology Unit, Department of Biomedical Sciences, Institute of Tropical Medicine, 2000 Antwerpen, Belgium; pdorny@itg.be

4 Center IDS, National Institute of Public Health and the Environment, RIVM, 3720 Bilthoven, The Netherlands; titia.kortbeek@rivm.nl

5 Institute of Acute Neurology, Academic Teaching Hospital Feldkirch, Carinagasse 47, 6800 Feldkirch, Austria; joachim.blocher@gmail.com

6 Center for Global Health, Department of Neurology, Technical University of Munich, 81675 Munich, Germany; veronika.schmidt@tum.de (V.S.); andrea.winkler@tum.de (A.S.W.)

7 Department of Veterinary Public Health and Food Safety, Faculty of Veterinary Medicine, Ghent University, 9820 Merelbeke, Belgium; Sarah.Gabriel@UGent.be

8 Centre for Global Health, Institute of Health and Society, University of Oslo, 0318 Oslo, Norway

* Correspondence: mariaangeles.gomezmorales@iss.it

$+\quad$ These authors equally contributed to this work.

‡ Ring Trial Participants: Claire Alexander, claire.alexander@ggc.scot.nhs.uk; Herbert Auer, herbert.auer@meduniwien.ac.at; Enikő Barabás, eniko.barabas@gmail.com; Silvana Belo, silvanabelo@ihmt.unl.pt; Clarissa Prazeres da Costa; clarissa.dacosta@tum.de; Zorica Dakic, zorda_dakic@yahoo.com; Maja Jurhar-Pavlova,jurharm@yahoo.com; Patricia Mihailescu, patriciamihailescu@yahoo.com; Elias Papadopoulos, eliaspap@vet.auth.gr; Maria Flores, mflores@externos.isciii.es; Maria J. Perteguer, chus.perteguer@isciii.es; Ingrid Reiter-Owona, reiter-owona@microbiology-bonn.de; Barbara Soba, barbara.soba@mf.uni-lj.si; Christen Rune Stensvold, run@ssi.dk.

Abstract: Laboratory tools for diagnosing taeniosis/cysticercosis in non-endemic countries are available; however, there is little data on their performance. To provide information on the sensitivity, specificity, and reproducibility of these tools, inter-laboratory studies were organized within the EU COST-Action CYSTINET (TD1302). Two serological and one coprological Ring Trials (RTs) were organized to test a panel of human-derived sera and stool samples using assays routinely conducted by the participating laboratories to detect Taenia spp. infections. Four Western blots (WBs) and five ELISAs were used by nine laboratories for cysticercosis diagnosis. In the first serological RT, the overall sensitivity was $67.6 \%$ (95\% CI, 59.1-75.4), whereas specificity was 97\% (95\% CI, 89.8-99.6). WBs recorded the best accuracy. A second serological RT was organized, to assess the three tests most frequently used during the first RT. Two out of six laboratories performed all the three tests. The overall sensitivity and specificity were 52.8\% (95\% CI, 42.8-62.7) and 98.1\% (95\% CI, 93.2-99.7), respectively. Laboratory performance strongly affected test results. Twelve laboratories participated in the coprological RT using conventional microscopy and six laboratories used molecular assays. Traditional diagnosis by microscopy yielded better results than molecular diagnosis. This may have been influenced by the lack of standardization of molecular tests across participating laboratories. 
Keywords: cysticercosis; neurocysticercosis; Taenia spp.; diagnosis; taeniosis; collaborative study; ring trial

\section{Introduction}

Human cysticercosis (CC) is a zoonotic infection caused by the metacestode larval stage (cysticercus) of the pork tapeworm Taenia solium. In humans, cysticerci can establish in the central nervous system (neurocysticercosis, NCC), eye, muscle, sub-cutaneous, and, in rare cases, in other tissues. NCC is considered the most common helminth infection of the nervous system and is a major cause of epilepsy in low-income endemic countries [1,2]. Human taeniosis, caused by the presence of the adult tapeworm of T. solium or the beef tapeworm Taenia saginata in the gut, is not associated with major clinical symptoms but has significant implications as it allows the perpetuation of the parasite life cycle. Taeniosis caused by T. solium, represents a risk of NCC in tapeworm carriers and for people who live within the same environment. T. saginata causes human taeniosis and important economic losses in the bovine meat sector due to condemnation of carcasses of infected cattle, its natural intermediate host, harboring cysticerci $[3,4]$. In contrast to T. solium, T. saginata does not cause human NCC. A third human Taenia sp., Taenia asiatica, never recorded in Europe either as an autochthonous or as an imported case [5], was not included in the present study.

Taenia solium is highly endemic in Latin America, Asia, and sub-Saharan Africa where poor sanitation and free-ranging pigs with access to human feces contribute to the life cycle [6-8]. In the European Union member states and associated countries (henceforth EU), T. solium was endemic in the past, although recent publications suggest that autochthonous cases may still be acquired in some regions [9-13]. In recent years, imported NCC cases have increased in parallel to increased migration and travel [14]. In 2014, T. solium was ranked by an international panel of experts as the food-borne parasite of greatest global concern, affecting millions of individuals every year and causing substantial economic impact [15]. In 2016 and considering Europe as a whole, T. solium was ranked tenth among 27 parasites; however, when individual European regions were considered, this parasite had a higher ranking in eastern Europe [13,16].

Clinical manifestations of NCC are pleomorphic, variable, and non-specific, being related to differences in the number, size, location, and state of the parasite (calcified vs. viable cysts) and to the severity of the host's immune response. Although no pathognomonic clinical picture exists, and in fact most patients remain asymptomatic for a long time, epileptic seizures and severe chronic progressive headaches are suggestive of NCC in endemic regions [17]. In non-endemic regions, the diagnosis of NCC is primarily based on neuroimaging, confirmed/aided by serology and a history of travel to or immigration from a $T$. solium endemic area $[7,18,19]$. The detection of taeniosis is most commonly made by microscopic examination of stool to detect eggs whose morphology and size are family specific (Taeniidae). Several in-house and commercial tools are used for the diagnosis of taeniosis/(neuro)cysticercosis by European clinical laboratories, [5]. However, data on their performance remains patchy. In order to address this issue, inter-laboratory collaborative studies (Ring Trials, RTs) were organized within the European Network on Taeniosis/Cysticercosis (CYSTINET) [20]. These studies aimed to determine the accuracy, sensitivity, specificity, and reproducibility of assays used by clinical laboratories in the detection of Taenia spp. infections in Europe.

\section{Materials and Methods}

\subsection{The European Network on Taeniosis/Cysticercosis: COST TD1302 Action CYSTINET}

CYSTINET [20] is a multidisciplinary group of highly motivated scientists with substantial research output and expertise. It is recognized by the international community as a network of excellence. Between 2014 and 2017, CYSTINET members increased from 55 
to over 150, representing 35 countries, and the group has remained active after the end of the COST grant period. The main objective of this Action was and still is to address the T. solium/T. saginata disease complexes from a One Health perspective through building a strong, extensive, multi-and interdisciplinary scientific network to induce sustainable collaborations and advance knowledge and understanding of taeniosis/cysticercosis. Specific objectives included the development of innovative and harmonized diagnostic and cost-efficient control tools, for which intra-European collaboration is essential to prevent the transmission of T. saginata and identify EU imported cases of taeniosis/cysticercosis caused by $T$. solium.

\subsection{Collaborative Studies}

During the COST TD1302 Action, CYSTINET members were invited via e-mail to express their interest in joining these collaborative studies. Participating laboratories are shown in Table 1. In the first RT, laboratories were invited to test a panel of human sera using their routine laboratory serological test/s, based on T. solium antibody or antigen detection. In the second RT, laboratories were invited to test a panel of human sera by the three assays that were determined by the first RT to be the most frequently utilized. In the third RT, laboratories were invited to test a panel of human stool by conventional microscopy (micro-coprological RT) and molecular (molecular-coprological RT) assays routinely used in their laboratories for the detection of Taenia infections (T. saginata/ T. solium).

Table 1. Laboratories and countries participating in the Ring Trials (RT).

\begin{tabular}{|c|c|c|c|c|c|}
\hline Participating Laboratories & Country & 1st RT & 2nd RT & $\begin{array}{l}\text { Micro-Copro } \\
\text { RT } \\
\end{array}$ & $\begin{array}{c}\text { Molecular-Copro } \\
\text { RT }\end{array}$ \\
\hline $\begin{array}{l}\text { Department of Medical Parasitology, Institute of } \\
\text { Specific Prophylaxis and Tropical Medicine, Center of } \\
\text { Pathophysiology, Infectiology and Immunology, } \\
\text { Medical University of Vienna, Vienna }\end{array}$ & Austria & yes & yes & yes & yes \\
\hline $\begin{array}{l}\text { Institute of Tropical Medicine (ITM), Department of } \\
\text { Biomedical Sciences, Veterinary Helminthology Unit, } \\
\text { Antwerp }\end{array}$ & Belgium & yes & yes & yes & yes \\
\hline $\begin{array}{c}\text { Laboratory of Parasitology, Statens Serum Institut, } \\
\text { Copenhagen }\end{array}$ & Denmark & no & no & yes & yes \\
\hline $\begin{array}{l}\text { Institute of Medical Microbiology, Immunology and } \\
\text { Parasitology, University Clinic Bonn, Bonn }\end{array}$ & Germany & yes & no & no & no \\
\hline $\begin{array}{l}\text { Institute of Medical Microbiology, Immunology and } \\
\text { Hygiene, Technical University of Munich }\end{array}$ & Germany & no & no & yes & no \\
\hline $\begin{array}{l}\text { Laboratory of Parasitology and Parasitic Diseases, } \\
\text { School of Veterinary Medicine, Faculty of Health } \\
\text { Sciences, Aristotle University, Thessaloniki }\end{array}$ & Greece & no & no & yes & no \\
\hline $\begin{array}{l}\text { Department of Infectious Diseases, Istituto Superiore } \\
\text { di Sanità, Rome }\end{array}$ & Italy & yes & yes & yes & yes \\
\hline $\begin{array}{l}\text { Institute of Microbiology and Parasitology, Medical } \\
\text { Faculty, University "Ss. Ciryl and Methodius", Skopje }\end{array}$ & $\begin{array}{l}\text { Republic of North } \\
\text { Macedonia }\end{array}$ & no & no & yes & no \\
\hline $\begin{array}{l}\text { Center IDS, National Institute of Public Health and the } \\
\text { Environment, RIVM, Bilthoven }\end{array}$ & Netherlands & yes & yes & yes & yes \\
\hline $\begin{array}{l}\text { Unidade de Parasitologia Médica, Instituto de Higiene } \\
\text { e Medicina Tropical; Universidade NOVA de Lisboa }\end{array}$ & Portugal & yes & no & no & no \\
\hline Mureic County Clinical Hospital, Mures & Romania & no & no & yes & no \\
\hline Eco-Para-Diagnostic Medical Center, Bucharest & Romania & no & no & yes & no \\
\hline $\begin{array}{l}\text { Parasitological Laboratory at Clinic for Infectious and } \\
\text { Tropical Diseases, Clinical Center of Serbia, Belgrade }\end{array}$ & Serbia & yes & no & no & no \\
\hline $\begin{array}{l}\text { Laboratory for Parasitology, Institute of Microbiology } \\
\text { and Immunology, Faculty of Medicine, University of } \\
\text { Ljubljana, Ljubljana }\end{array}$ & Slovenia & yes & yes & yes & no \\
\hline
\end{tabular}


Table 1. Cont.

\begin{tabular}{cccccc}
\hline Participating Laboratories & Country & 1st RT & 2nd RT & $\begin{array}{c}\text { Micro-Copro } \\
\text { RT }\end{array}$ & $\begin{array}{c}\text { Molecular-Copro } \\
\text { RT }\end{array}$ \\
\hline $\begin{array}{c}\text { Instituto de Salud Carlos III, Centro Nacional de } \\
\text { Microbiología Parasitologia, Majadahonda, Madrid }\end{array}$ & Spain & yes & yes & yes & yes \\
\hline SPDRL Glasgow Royal Infirmary, Glasgow & UK & yes & no & no & no \\
\hline
\end{tabular}

\subsection{Panels of Samples}

CYSTINET members provided serum and fecal samples to the European Union Reference Laboratory for Parasites (EURLP), Rome, Italy. These samples had been collected from confirmed patients following informed consent for their use in clinical studies and were stored under the required conditions $\left(-20^{\circ} \mathrm{C}\right.$ or $\left.-80^{\circ} \mathrm{C}\right)$ in respective laboratories for a number of years [21,22]. Upon arrival at the EURLP, samples were coded, and aliquots were sent out to the participating laboratories for blind analyses. Panels of samples for the two serological RTs are shown in Table 2.

Table 2. Panels of human serum samples provided for the Ring Trials (RT).

\begin{tabular}{|c|c|c|c|}
\hline & Sample Code & Sample Origin & Location of the Lesion \\
\hline \multirow[t]{12}{*}{ 1st RT } & 1 & Italian male with cystic echinococcosis & Liver \\
\hline & 2 & Cape Verdean male with NCC & $\mathrm{I}^{1}$ \\
\hline & 3 & Italian female travelling in South America with NCC & $\mathrm{E}^{2}$ \\
\hline & 4 & Italian blood donor & $\mathrm{NA}^{3}$ \\
\hline & 5 & Dutch female travelling in Asia with NCC & I \\
\hline & 6 & Indian female with NCC & I \\
\hline & 7 & Latin American male with NCC & $\mathrm{E}$ \\
\hline & 8 & Mexican male with NCC & $\mathrm{I}$ \\
\hline & 9 & Italian blood donor & NA \\
\hline & 10 & Mexican male with NCC & $\mathrm{I}$ \\
\hline & 11 & Cuban female with adenocarcinoma & NA \\
\hline & 12 & Peruvian male with NCC & I \\
\hline \multirow[t]{16}{*}{ 2nd RT } & 1 & Italian male with trichinellosis & NA \\
\hline & 2 & Italian male with cystic echinococcosis & Liver \\
\hline & 3 & Italian male with opisthorchiasis & Biliary ducts \\
\hline & 4 & Italian female travelling in South America with NCC & $\mathrm{E}$ \\
\hline & 5 & Italian blood donor & NA \\
\hline & 6 & Italian blood donor & NA \\
\hline & 7 & Zambian male with NCC & $\mathrm{U}^{4}$ \\
\hline & 8 & Zambian male with NCC & $\mathrm{U}$ \\
\hline & 9 & Italian female living in Mexico with NCC & $\mathrm{U}$ \\
\hline & 10 & Zambian male with NCC & $\mathrm{U}$ \\
\hline & 11 & Zambian male with NCC & $\mathrm{U}$ \\
\hline & 12 & South American male with NCC & $\mathrm{U}$ \\
\hline & 13 & Italian male with trichinellosis & NA \\
\hline & 14 & Italian male with cystic echinococcosis & NA \\
\hline & 15 & Italian male with opisthorchiasis & Biliary ducts \\
\hline & 16 & Italian male with NCC & $\mathrm{U}$ \\
\hline
\end{tabular}

${ }^{1}$ intra-parenchymal; ${ }^{2}$ extra-parenchymal; ${ }^{3}$ not applicable; ${ }^{4}$ unknown.

A total of 12 serum samples, eight of which were from patients presenting clinical signs and symptoms suggestive of NCC, confirmed by cerebral computed tomography (CT) were used for the first serological RT. Of these 12, two were from healthy Italian individuals (used as negative reference samples), who, according to Italian law, are considered suitable for blood donation; one serum sample was from an Italian male with serological and ultrasound-confirmed cystic echinococcosis of the liver and one serum sample was from a 45-year-old Cuban female with cervical adenocarcinoma. Serum samples from NCC patients included in the first RT were categorized into two groups according to the localization of the cyst/s (intra-parenchymal or extra-parenchymal NCC) [23]. For the second 
serological RT, a panel of 16 samples was used, eight of which were from patients presenting clinical signs and symptoms suggestive of NCC confirmed by cerebral CT; however, data regarding cyst localization were not available for all samples. Six serum samples were from patients infected with other helminths, namely, Trichinella spiralis $(n=2)$, Echinococcus granulosus $(n=2)$, and Opisthorchis felineus $(n=2)$. Two serum samples were from Italian individuals considered suitable for blood donation. A total of five stool samples fixed in 95\% ethanol were used for the third RT (microscopic and molecular-coprological RTs) (Table 3). The panel for microscopy testing, included two samples from healthy donors which served as negative controls, one sample from a healthy donor spiked with a known burden of E. granulosus eggs $\left(1.5 \times 10^{3}\right.$ eggs/g of feces) and two samples from a confirmed T. saginata patient. One of these samples had a known T. saginata egg burden $\left(10^{3} \mathrm{eggs} / \mathrm{g}\right.$ of feces) and the second was spiked with egg-laden T. saginata verified proglottids. Samples 21,22 , and 23 from this panel should be classified positive for Taeniid eggs. In addition, for sample 23 the species identification could be reported (Table 3). The molecular-coprological RT panel included 3 samples from a healthy donor each spiked with $8 \mathrm{ng}$ of T. solium, T. saginata, and E. granulosus verified DNA. A fourth sample was from a confirmed T. solium patient spiked with egg-laden T. solium verified proglottids. A fifth sample from a healthy donor completed the panel and served as a negative control. For this panel, samples 25, 28, and 29 should be classified positive (Table 3).

Table 3. Panel of stool samples provided for the coprological Ring Trials.

\begin{tabular}{ccc}
\hline Test & Code & Sample Origin \\
\hline Microscopic & 20 & Healthy donor \\
& 21 & Taenia saginata infected patient $\left(10^{3}\right.$ eggs $\left./ \mathrm{g}\right)$ \\
& 22 & Healthy donor stool spiked with \\
& 23 & fixed Echinococcus granulosus eggs $\left(1.5 \times 10^{3}\right.$ eggs $\left./ \mathrm{g}\right)$ \\
Molecular & 24 & T. saginata infected patient (one mature proglottid) \\
& 25 & Healthy donor \\
& 26 & Healthy donor stool spiked with Taenia solium DNA (8 ng) \\
& 27 & Healthy donor \\
& 28 & Healthy donor stool spiked with E. granulosus DNA(8 ng) \\
& 29 & Healthy donor stool spiked with T. saginata DNA (8 ng) \\
& T. solium infected patient (one proglottid) \\
\hline
\end{tabular}

\subsection{Instructions to Participating Laboratories}

Frozen serum samples stored in dry ice and fecal samples refrigerated using ice blocks, were dispatched to the participating laboratories by an international courier. Each laboratory received four forms as follows: 1 . "Package check" to verify the content and the condition of the RT samples at arrival; 2. "Instruments and Materials" needed to perform the assays; 3." Procedure" step by step description of the assay/s used by each laboratory; and 4. "Results" to record the result obtained for each sample. A confidential code was assigned to each laboratory, which was sent by email on the day of package shipment.

\subsection{Data Analysis}

For serological and micro-coprological RTs, accuracy (or the degree of conformity of a measure to a true value; on the receiver-operator characteristic (ROC) curves, the accuracy is given by the area under the curve (AUC); sensitivity (the measure of how well a test can identify true positives); specificity (the measure of how well a test can identify true negatives); and inter-rater agreement (i.e., the degree of agreement among laboratories) were calculated using Stata v. 10 (StataCorp LLC, Lakeway Drive College Station, Texas, USA). Test agreement was expressed as kappa (K) index values. The scale of this measure of agreement ranges between ' 0 ' when the level of agreement is what would be expected to be observed by chance and ' 1 ', which indicates perfect agreement. For intermediate $\mathrm{K}$ index values, the following interpretations of agreement were used: 0.00-0.20: slight; $0.21-0.40$ : fair; $0.41-0.60$ : moderate; $0.61-0.80$ : substantial; and $0.81-1.00$ : 
almost perfect [24]. For molecular-coprological RT, diagnostic performance, i.e., sensitivity, specificity, and reliability, by test was calculated.

\section{Results}

\subsection{First Serological RT}

Ten laboratories participated in the first serological RT (Table 1). All laboratories but one received the panel of samples within $48 \mathrm{~h}$ of dispatch; the remaining laboratory received the samples four days after shipping. At delivery, the internal package temperature was in the range of $+1.9{ }^{\circ} \mathrm{C}--50^{\circ} \mathrm{C}$. The time between the arrival of the package at the laboratories and package control was less than $30 \mathrm{~min}$. During this time, packages were stored at $+4{ }^{\circ} \mathrm{C}$. Samples were to be tested soon after the initial control check.

For antibody detection, 11 tests were performed by nine laboratories. One laboratory was unable to obtain the kit used in their routine testing and therefore did not participate. Some laboratories used more than one assay (Table 4).

Table 4. Serological tests and number of tested samples performed by the laboratories in the course of the first and second Ring Trials (RT).

\begin{tabular}{|c|c|c|c|c|c|c|c|c|c|c|c|c|}
\hline \multirow{2}{*}{$\begin{array}{c}\text { 1st RT } \\
\text { Test }\end{array}$} & \multirow{2}{*}{$\begin{array}{c}\text { Assays } \\
\text { Antibody Detection }\end{array}$} & \multicolumn{11}{|c|}{ Laboratories (Code) } \\
\hline & & A & B & $\mathrm{C}$ & D & $\mathbf{E}$ & $\mathbf{F}$ & G & $\mathbf{H}$ & $\mathbf{J}$ & $\mathbf{L}$ & Total \\
\hline & & \multicolumn{11}{|c|}{ No. of Tested Sera } \\
\hline \multirow[t]{5}{*}{ ELISA } & Taenia solium IgG (Bioactiva Diagnostic ${ }^{\circledR}$, Bad Homburg, Germany) & 0 & 0 & 0 & 0 & 0 & 0 & 0 & 0 & 12 & 0 & 12 \\
\hline & Cysticercosis Antibody Kit (Cypress Diagnostics ${ }^{\circledR}$, Hulshoult, Belgium) & 0 & 0 & 0 & 0 & 0 & 0 & 12 & 0 & 0 & 0 & 12 \\
\hline & Enzyme-Linked ImmunoSorbent Assay (GST-T24H, in house) [25] & 0 & 0 & 12 & 0 & 0 & 0 & 0 & 0 & 0 & 0 & 12 \\
\hline & $\begin{array}{l}\text { Novagnost Taenia solium IgG (Novagnost }{ }^{\circledR} \text {, NovaTec } \\
\text { Immunodiagnostica GmbH, Dietzenbach, Germany) }\end{array}$ & 12 & 0 & 0 & 0 & 0 & 0 & 0 & 0 & 0 & 0 & 12 \\
\hline & $\begin{array}{l}\text { NovaLisa Taenia solium IgG ELISA (NovaLisa }{ }^{\circledR} \text {, Novatec } \\
\text { Immundiagnostica GmbH, Dietzenbach, Germany) }\end{array}$ & 0 & 0 & 12 & 0 & 0 & 0 & 0 & 12 & 0 & 0 & 24 \\
\hline \multirow[t]{4}{*}{ Western blot } & $\begin{array}{l}\text { Lentil lectin-bound glycoprotein enzyme-linked immunotransferblot } \\
\text { assay (LLGP-EITB) [26] }\end{array}$ & 0 & 12 & 12 & 0 & 0 & 0 & 0 & 0 & 0 & 12 & 36 \\
\hline & r-EITB [27] & 0 & 12 & 0 & 0 & 0 & 0 & 0 & 0 & 0 & 0 & 12 \\
\hline & r-T24H EITB [25] & 0 & 0 & 12 & 0 & 0 & 0 & 0 & 0 & 0 & 0 & 12 \\
\hline & CYSTICERCOSIS WB IgG (LDBIO Diagnostics ${ }^{\circledR}$, Lyon, France) & 0 & 12 & 0 & 12 & 0 & 12 & 0 & 12 & 0 & 0 & 48 \\
\hline \multirow[t]{3}{*}{ Other } & Counter immune-electrophoresis (CIE in house) & 0 & 0 & 0 & 0 & 0 & 0 & 0 & 0 & 12 & 0 & 12 \\
\hline & Immunofluorescent Assay (IFA in house) & 0 & 0 & 0 & 0 & 0 & 12 & 0 & 0 & 0 & 0 & 12 \\
\hline & 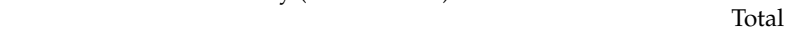 & 12 & 36 & 48 & 12 & 0 & 24 & 12 & 24 & 24 & 12 & 204 \\
\hline Test & Antigen detection & & & & & & & & & & & \\
\hline \multirow[t]{3}{*}{ ELISA } & Antigen-ELISA kit (ApDia ${ }^{\circledR}$, Turnhout, Belgium) & 0 & 12 & 12 & 0 & 0 & 0 & 0 & 0 & 0 & 0 & 24 \\
\hline & Double antibodies ELISA (DAET in house) [28] & 0 & 0 & 0 & 0 & 12 & 0 & 0 & 0 & 0 & 0 & 12 \\
\hline & 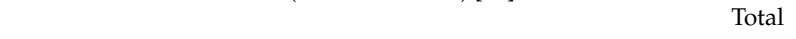 & 0 & 12 & 12 & 0 & 12 & 0 & 0 & 0 & 0 & 0 & 36 \\
\hline \multirow{6}{*}{$\begin{array}{l}\text { 2nd RT } \\
\text { Test }\end{array}$} & & & & & & & & & & & & \\
\hline & Antibody detection & & & & & & & & & & & \\
\hline & $\begin{array}{l}\text { Lentil lectin-bound glycoprotein enzyme-linked immunotransferblot } \\
\text { assay (LLGP-EITB) [26] }\end{array}$ & 0 & 16 & 16 & 0 & 0 & 0 & 0 & 0 & 0 & 16 & 48 \\
\hline & CYSTICERCOSIS WB IgG-(LDBIO Diagnostics ${ }^{\circledR}$, Lyon, France) & 0 & 16 & 16 & 16 & 16 & 0 & 0 & 16 & 0 & 16 & 96 \\
\hline & $\begin{array}{l}\text { NovaLisa Taenia solium IgG-ELISA (NovaLisa }{ }^{\circledR} \text {, Novatec } \\
\text { Immundiagnostica GmbH, Dietzenbach, Germany) }\end{array}$ & 0 & 0 & 16 & 0 & 16 & 0 & 0 & 16 & 0 & 16 & 64 \\
\hline & 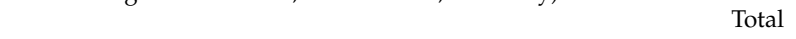 & 0 & 32 & 48 & 16 & 32 & 0 & 0 & 32 & 0 & 48 & 208 \\
\hline
\end{tabular}

The combined overall diagnostic performance of the tests used across the participating laboratories were as follows: sensitivity, $67.6 \%$ (95\% CI, 59.1-75.4); specificity, 97\% (95\% CI, 89.8-99.6); accuracy, $77.4 \%$ (95\% CI, 71.2-82.99). The results for sensitivity, specificity, reliability, and accuracy by test are shown in Table 5.

LLGP-EITB [26] and Cypress Diagnostics ${ }^{\circledR}$ (Hulshoult, Belgium) showed the highest accuracy, i.e., 0.96 (95\% CI 0.85-0.99) and 0.94 (95\% CI 0.61-0.99), respectively. Considering both laboratory and test, the highest AUC (accuracy) was recorded for LLGP-EITB [26] performed by laboratory C (1.0, 95\% CI 0.73-1.00; Table 6). 
Table 5. Diagnostic accuracy by test of the first and second Ring Trials (RT).

\begin{tabular}{|c|c|c|c|c|c|c|c|c|c|c|}
\hline $\begin{array}{l}\text { First RT } \\
\text { Antibody Detection Test }\end{array}$ & $\mathrm{TN}^{1}$ & $\mathrm{FP}^{2}$ & $\mathrm{FN}^{3}$ & $\mathrm{TP}^{4}$ & $\begin{array}{l}\text { Total } \\
\text { Samples }\end{array}$ & $\begin{array}{l}\text { Sensitivity } \\
(\%)\end{array}$ & $\begin{array}{l}\text { Specificity } \\
\text { (\%) }\end{array}$ & $\begin{array}{l}\text { Correctly Classified } \\
\text { (Reliability) } \\
\text { (\%) }\end{array}$ & $\begin{array}{l}\text { ROC Area } \\
\text { (Accuracy) }\end{array}$ & $95 \% \mathrm{CI}$ \\
\hline Bioactiva Diagnostica ${ }^{\circledR}$ (Bad Homburg, Germany) & 4 & 0 & 3 & 5 & 12 & 62.5 & 100 & 75.0 & 0.8125 & $0.51-0.97$ \\
\hline Cypress Diagnostics ${ }^{\circledR}$ (Hulshoult, Belgium) & 4 & 0 & 1 & 7 & 12 & 87.5 & 100 & 91.7 & 0.9375 & $0.61-0.99$ \\
\hline GTS-T24H [25] & 4 & 0 & 2 & 6 & 12 & 75.0 & 100 & 83.3 & 0.8750 & $0.61-0.99$ \\
\hline $\begin{array}{l}\text { Novagnost }{ }^{\circledR} \text { (NovaTec Immunodiagnostica GmbH, } \\
\text { Dietzenbach, Germany) }\end{array}$ & 4 & 0 & 5 & 3 & 12 & 37.5 & 100 & 58.3 & 0.6875 & $0.35-0.90$ \\
\hline $\begin{array}{l}\text { NovaLisa }^{\circledR} \text { (Novatec Immundiagnostica GmbH, } \\
\text { Dietzenbach, Germany) }\end{array}$ & 7 & 1 & 6 & 10 & 24 & 62.5 & 87.5 & 70.8 & 0.7500 & $0.53-0.90$ \\
\hline r-EITB [27] & 4 & 0 & 2 & 6 & 12 & 75.0 & 100 & 83.3 & 0.8750 & $0.61-0.99$ \\
\hline r-T24H EITB [25] & 4 & 0 & 3 & 5 & 12 & 62.5 & 100 & 75.0 & 0.8125 & $0.51-0.97$ \\
\hline LDBIO Diagnostics $^{\circledR}$ (Lyon, France) & 16 & 0 & 10 & 22 & 48 & 68.8 & 100 & 79.2 & 0.8438 & $0.72-0.93$ \\
\hline CIE & 4 & 0 & 8 & 0 & 12 & 0 & 100 & 33.3 & $\mathrm{NE}^{5}$ & NE \\
\hline IFA & 4 & 0 & 2 & 6 & 12 & 75.0 & 100 & 83.3 & 0.8750 & $0.61-0.99$ \\
\hline \multicolumn{11}{|l|}{ Antigen detection test } \\
\hline ApDia $^{\circledR}$ (Turnhout, Belgium) & 7 & 1 & 9 & 7 & 24 & 43.7 & 87.5 & 58.3 & 0.66 & $0.45-0.84$ \\
\hline DAET [28] & 4 & 0 & 3 & 5 & 12 & 62.5 & 100 & 75.0 & 0.81 & $0.51-0.99$ \\
\hline \multicolumn{11}{|l|}{$\begin{array}{l}\text { Second RT } \\
\text { Antibody detection test }\end{array}$} \\
\hline LLGP-EITB [26] & 23 & 1 & 8 & 16 & 48 & 66.67 & 95.83 & 81.25 & 0.8125 & $0.67-0.91$ \\
\hline LDBIO Diagnostics $^{\circledR}$ (Lyon, France) & 47 & 1 & 20 & 28 & 96 & 58.33 & 97.92 & 78.13 & 0.7812 & $0.65-0.86$ \\
\hline $\begin{array}{l}\text { NovaLisa }{ }^{\circledR} \text { (Novatec Immundiagnostica GmbH, } \\
\text { Dietzenbach, Germany) }\end{array}$ & 32 & 0 & 21 & 11 & 64 & 34.38 & 100 & 67.19 & 0.6719 & $0.54-0.78$ \\
\hline
\end{tabular}

${ }^{1}$ True negative; ${ }^{2}$ false positive; ${ }^{3}$ false negative; ${ }^{4}$ true positive; ${ }^{5}$ not evaluable. 
Table 6. Diagnostic accuracy by laboratory and test of the first and second Ring Trials (RT).

\begin{tabular}{|c|c|c|c|c|c|c|c|c|c|c|c|}
\hline $\begin{array}{l}\text { 1st RT } \\
\text { Antibody Detection Test }\end{array}$ & $\begin{array}{l}\text { Lab } \\
\text { Code }\end{array}$ & $\mathrm{TN}^{1}$ & $\mathbf{F P}^{2}$ & $\mathrm{FN}^{3}$ & $\mathrm{TP}^{4}$ & $\begin{array}{c}\text { Total } \\
\text { Samples }\end{array}$ & $\begin{array}{l}\text { Sensitivity } \\
(\%)\end{array}$ & $\begin{array}{l}\text { Specificity } \\
\quad(\%)\end{array}$ & $\begin{array}{c}\text { Correctly Classified } \\
\text { (Reliability)(\%) }\end{array}$ & $\begin{array}{l}\text { ROC Area } \\
\text { (Accuracy) }\end{array}$ & $95 \%$ CI \\
\hline \multirow{3}{*}{ LLGP-EITB [26] } & B & 4 & 0 & 1 & 7 & 12 & 87.5 & 100 & 91.7 & 0.9375 & $0.61-0.99$ \\
\hline & $\mathrm{C}$ & 4 & 0 & 0 & 8 & 12 & 100 & 100 & 100 & 1.0 & $0.73-1.0$ \\
\hline & $\mathrm{L}$ & 4 & 0 & 1 & 7 & 12 & 87.5 & 100 & 91.7 & 0.9375 & $0.61-0.99$ \\
\hline \multirow{4}{*}{ LDBIO Diagnostics ${ }^{\circledR}$ (Lyon, France) } & B & 4 & 0 & 3 & 5 & 12 & 62.5 & 100 & 75.0 & 0.8125 & $0.51-0.97$ \\
\hline & $\mathrm{D}$ & 4 & 0 & 2 & 6 & 12 & 75.0 & 100 & 83.3 & 0.875 & $0.61-0.99$ \\
\hline & $\mathrm{F}$ & 4 & 0 & 2 & 6 & 12 & 75.0 & 100 & 83.3 & 0.875 & $0.61-0.99$ \\
\hline & $\mathrm{H}$ & 4 & 0 & 3 & 5 & 12 & 62.5 & 100 & 75.0 & 0.8125 & $0.51-0.97$ \\
\hline NovaLisa ${ }^{\circledR}$ (Novatec Immundiagnostica GmbH, & $\mathrm{C}$ & 3 & 1 & 3 & 5 & 12 & 62.5 & 75.0 & 66.7 & 0.6875 & $0.35-0.90$ \\
\hline Dietzenbach, Germany) & $\mathrm{H}$ & 4 & 0 & 3 & 5 & 12 & 62.5 & 100 & 75.0 & 0.8125 & $0.51-0.98$ \\
\hline \multicolumn{12}{|l|}{ Antigen detection test } \\
\hline \multirow{2}{*}{ apDia $^{\circledR}$ (Turnhout, Belgium) } & B & 4 & 0 & 5 & 3 & 12 & 35.0 & 100 & 58.3 & 0.69 & $0.35-0.90$ \\
\hline & $\mathrm{C}$ & 3 & 1 & 4 & 4 & 12 & 50.0 & 75.0 & 58.3 & 0.62 & $0.35-0.90$ \\
\hline \multicolumn{12}{|l|}{$\begin{array}{l}\text { 2nd RT } \\
\text { Antibody detection test }\end{array}$} \\
\hline \multirow[t]{5}{*}{ LLGP-EITB [26] } & B & 7 & 1 & 4 & 4 & 16 & 50.0 & 87.5 & 68.75 & 0.687 & $0.42-0.89$ \\
\hline & $\mathrm{C}$ & 8 & 0 & 2 & 6 & 16 & 75.0 & 100 & 87.5 & 0.875 & $0.62-0.98$ \\
\hline & $\mathrm{L}$ & 8 & 0 & 2 & 6 & 16 & 75.0 & 100 & 87.5 & 0.875 & $0.62-0.98$ \\
\hline & $\mathrm{B}$ & 7 & 1 & 4 & 4 & 16 & 50.0 & 87.5 & 68.75 & 0.687 & $0.42-0.89$ \\
\hline & $\mathrm{C}$ & 8 & 0 & 1 & 7 & 16 & 87.5 & 100 & 93.75 & 0.937 & $0.69-0.99$ \\
\hline \multirow{4}{*}{ LDBIO Diagnostics $^{\circledR}$ (Lyon, France) } & $\mathrm{D}$ & 8 & 0 & 4 & 4 & 16 & 50.0 & 100 & 75.0 & 0.75 & $0.47-0.92$ \\
\hline & $\mathrm{E}$ & 8 & 0 & 4 & 4 & 16 & 50.0 & 100 & 75.0 & 0.75 & $0.47-0.92$ \\
\hline & $\mathrm{H}$ & 8 & 0 & 4 & 4 & 16 & 50.0 & 100 & 75.0 & 0.75 & $0.47-0.92$ \\
\hline & $\mathrm{L}$ & 8 & 0 & 3 & 5 & 16 & 62.5 & 100 & 81.25 & 0.812 & $0.54-0.95$ \\
\hline \multirow{4}{*}{$\begin{array}{l}\text { NovaLisa }{ }^{\circledR} \text { (Novatec Immundiagnostica GmbH, } \\
\text { Dietzenbach, Germany) }\end{array}$} & C & 8 & 0 & 5 & 3 & 16 & 37.5 & 100 & 68.75 & 0.687 & $0.41-0.89$ \\
\hline & $\mathrm{E}$ & 8 & 0 & 6 & 2 & 16 & 25.0 & 100 & 62.5 & 0.625 & $0.35-0.85$ \\
\hline & $\mathrm{H}$ & 8 & 0 & 5 & 3 & 16 & 37.5 & 100 & 68.75 & 0.687 & $0.41-0.89$ \\
\hline & $\mathrm{L}$ & 8 & 0 & 5 & 3 & 16 & 37.5 & 100 & 68.75 & 0.687 & $0.41-0.89$ \\
\hline
\end{tabular}

${ }^{1}$ True negative; ${ }^{2}$ false positive; ${ }^{3}$ false negative; ${ }^{4}$ true positive. 
All tests but one were $100 \%$ specific, with only sample coded 11 testing falsely positive by NovaLisa ${ }^{\circledR}$ (Novatec Immundiagnostica GmbH, Dietzenbach, Germany) in laboratory $\mathrm{C}$ (Tables 5 and 6). Focusing only on false negative results (18 samples), multilevel logistic analysis showed that the Odds Ratio (OR) for LLGP-EITB [26] vs. LDBIO Diagnostics ${ }^{\circledR}$ (Lyon, France ) was $0.2\left(p<0.05,95 \%\right.$ CI 0.04-1.02), NovaLisa ${ }^{\circledR}$ (Novatec Immundiagnostica $\mathrm{GmbH}$, Dietzenbach, Germany) vs. LLGP-EITB [26] was 6.6 ( $p<0.036,95 \%$ CI 1.128-38.60), and NovaLisa ${ }^{\circledR}$ (Novatec Immundiagnostica GmbH, Dietzenbach, Germany) vs. LDBIO Diagnostics ${ }^{\circledR}$ (Lyon, France) was 1.32 ( $p<0.66,95 \%$ CI 0.375-4.645). The inter-rater agreement among laboratories performing the same test is shown in Table 7.

The $\mathrm{K}$ index value for laboratories using LLGP-EITB [26] and LDBIO Diagnostics ${ }^{\circledR}$ (Lyon, France) indicated a good level of agreement. A total of $39.2 \%$ and $11.8 \%$ of samples from individuals with intra-parenchymal and extra-parenchymal lesions, respectively, were classified as negative (false negative, Table 8).

Moreover, multilevel logistic analysis adjusted by laboratory (random effect) and adjusted by test (fixed effect) and laboratory (random effect), showed higher association for the intra-parenchymal localization of cysts with false negatives than the extra-parenchymal localization (Table 9).

For antigen detection, two tests were performed by three laboratories (Table 4). The highest accuracy was reported for an in-house ELISA used by laboratory E (DAET [28], AUC 0.81, 95\% CI 0.51-0.99; Table 5). A similar accuracy was recorded by laboratory B (AUC 0.69, 95\% CI 0.35-0.90) and laboratory C (AUC 0.62, 95\% CI 0.35-0.90) using the commercial kit apDia ${ }^{\circledR}$ (Turnhout, Belgium), with a K index value of 0.66 (95\% CI 0.2-1.0; Tables 6 and 7).

Table 7. Inter-rater agreement of serological Ring Trials (RT).

\begin{tabular}{|c|c|c|c|}
\hline $\begin{array}{c}\text { 1st RT } \\
\text { Antibody Detection Test (Laboratory Code) }\end{array}$ & $\mathbf{K}$ & $95 \% \mathrm{CI}$ & $p$ \\
\hline LLGP-EITB [26] (B, C, L) & 0.88 & $0.72-1.00$ & $<0.001$ \\
\hline LDBIO Diagnostics $^{\circledR}$ (Lyon, France )(B, D, F, H) & 0.89 & $0.88-1.00$ & $<0.001$ \\
\hline $\begin{array}{c}\text { NovaLisa }^{\circledR} \text { (Novatec Immundiagnostica GmbH, } \\
\text { Dietzenbach, Germany) }(\mathrm{C}, \mathrm{H})\end{array}$ & 0.50 & $0.02-0.98$ & 0.04 \\
\hline \multicolumn{4}{|l|}{ Antigen detection test } \\
\hline $\operatorname{apDia}^{\circledR}($ Turnhout, Belgium $)(B, C)$ & 0.66 & $0.20-1.00$ & - \\
\hline \multicolumn{4}{|l|}{$\begin{array}{l}\text { 2nd RT } \\
\text { Antibody detection test }\end{array}$} \\
\hline LLGP-EITB [26] (B, C, L) & 0.36 & & 0.006 \\
\hline $\begin{array}{c}\text { LDBIO Diagnostics }^{\circledR} \text { (Lyon, France) }(\mathrm{B}, \mathrm{C}, \mathrm{D}, \mathrm{E}, \\
\mathrm{H}, \mathrm{L})\end{array}$ & 0.55 & & 0.000 \\
\hline $\begin{array}{c}\text { NovaLisa }{ }^{\circledR} \text { (Novatec Immundiagnostica GmbH, } \\
\text { Dietzenbach, Germany) (C, E, H, L) }\end{array}$ & 0.89 & & 0.000 \\
\hline
\end{tabular}

Table 8. Influence of the cyst localization on the results of the first serological Ring Trial.

\begin{tabular}{cccc}
\hline False Negative & Intra-Parenchymal & Extra-Parenchymal & Total \\
\hline N (\%) & N (\%) & 92 \\
No & 62 & 30 & 44 \\
Yes & $40(39.2)$ & $4(11.8)$ & 136 \\
Total & 102 & 34 & \\
\hline
\end{tabular}


Table 9. Odds Ratio (OR) of the intra-parenchymal versus extra-parenchymal cysts on the serological results of the first Ring Trial.

\begin{tabular}{lccc}
\hline \multicolumn{1}{c}{ False Negative } & OR & 95\% CI ( $p$-Value) & Note \\
\hline $\begin{array}{l}\text { Intra-parenchymal vs. extra } \\
\text { parenchymal }\end{array}$ & 5.45 & $1.70-17.51(0.004)$ & adjusted by laboratory (random effect) \\
$\begin{array}{l}\text { Intra-parenchymal vs. extra } \\
\text { parenchymal }\end{array}$ & 9.99 & $2.11-47.21(0.004)$ & adjusted by test (fixed effect) and laboratory (random effect) \\
\hline
\end{tabular}

\subsection{Second Serological RT}

All six participating laboratories (Table 1) received the package within $24 \mathrm{~h}$ from dispatch and upon arrival all serum samples were still frozen. Only two laboratories tested the panel of serum samples by the three requested assays (LLGP-EITB [26], LDBIO Diagnostics ${ }^{\circledR}$ (Lyon, France), and NovaLisa ${ }^{\circledR}$ (Novatec Immundiagnostica GmbH, Dietzenbach, Germany); two laboratories tested the samples by LDBIO Diagnostics ${ }^{\circledR}$ (Lyon, France) and NovaLisa ${ }^{\circledR}$ Novatec Immundiagnostica GmbH, Dietzenbach, Germany); one by LLGPEITB [26] and LDBIO Diagnostics ${ }^{\circledR}$ (Lyon, France); and one by LDBIO Diagnostics ${ }^{\circledR}$ (Lyon, France) (Table 4). Considering all the outputs of the six laboratories and the three tests together, sensitivity was 52.8\% (95\% CI 42.8-62.7), specificity $98.1 \%$ (95\% CI 93.2-99.7), and accuracy $75.5 \%$ (95\% CI 69.1-81.17). The most accurate test was LLGP-EITB [26] (AUC 0.8125, 95\% CI 0.67-0.91; Table 5). Considering tests and laboratories, the highest AUC value was recorded for the LDBIO Diagnostics ${ }^{\circledR}$ (Lyon, France) assay performed by laboratory C (AUC 0.94, 95\% CI 0.69-0.99). One sample (sample 15) tested erroneously positive by two assays performed at the same laboratory (Table 6). When considering only false negatives, the multilevel logistic analysis showed that OR for LLGP-EITB [26] vs. LDBIO Diagnostics ${ }^{\circledR}$ (Lyon, France) was $0.7\left(p<0.495,95 \%\right.$ CI 0.251-1.95) and NovaLisa ${ }^{\circledR}$ (Novatec Immundiagnostica GmbH, Dietzenbach, Germany) vs. LLGP-EITB [26] was 3.82 ( $p<0.02,95 \%$ CI 1.24-11.69). The inter-rater agreement among laboratories performing the same test is shown in Table 7.

\subsection{Third RT (Microscopic and Molecular-Coprological RTs)}

Stool samples were sent refrigerated, and all participating laboratories received the package within $24 \mathrm{~h}$. At arrival, the temperature inside the parcel was less than $15^{\circ} \mathrm{C}$. Twelve laboratories representing 11 countries participated in the microscopy RT (Table 1). Saline and iodine wet mount preparations of fecal smears for direct examination were carried out by seven laboratories, microscopic examination after concentration (whether by sedimentation or flotation) was performed in 11 laboratories, and Kato-Katz followed by Ziehl-Neelsen staining [29] was carried out in one laboratory (Table 10).

Eleven (91.7\%) out of twelve laboratories correctly identified all the samples (Table S1). The highest accuracy was recorded for the direct examination of fecal smears after iodine and wet mount preparations (AUC 1.0, 95\% CI 0.99-1.0, Table 11). All laboratories but one correctly identified Taeniid eggs in the three known positive microscopy RT samples. In contrast, T. saginata proglottids were recovered by only 4 (33\%) laboratories. Of the 12 participating laboratories $91.7 \%$ correctly identified the negative controls, whereas a false positive was recorded by a single laboratory. 
Table 10. Number of fecal samples tested by each laboratory per assay.

\begin{tabular}{|c|c|c|c|c|c|c|c|c|c|c|c|c|c|}
\hline \multirow{2}{*}{ Micro-Copro Ring Trial Test } & \multicolumn{13}{|c|}{ Laboratory Code } \\
\hline & B & $\mathrm{C}$ & D & $\mathrm{E}$ & $\mathbf{F}$ & $\mathbf{H}$ & $\mathbf{L}$ & $\mathbf{R}$ & $\mathrm{S}$ & $\mathbf{W}$ & $\mathbf{Y}$ & $\mathbf{Z}$ & Total \\
\hline & \multicolumn{13}{|c|}{$\mathrm{N}^{\mathrm{o}}$ of fecal samples } \\
\hline $\begin{array}{l}\text { Saline and iodine wet mount preparations of fecal } \\
\text { smears for direct examination }\end{array}$ & 5 & 5 & 0 & 0 & 0 & 5 & 5 & 0 & 0 & 5 & 5 & 5 & 35 \\
\hline $\begin{array}{l}\text { Microscopic examination after concentration } \\
\text { (whether by sedimentation or flotation) }\end{array}$ & 5 & 5 & 5 & 5 & 5 & 5 & 5 & 5 & 5 & 5 & 5 & 0 & 55 \\
\hline Kato-Katz followed by Ziehl-Neelsen staining [29] & 0 & 0 & 0 & 0 & 0 & 0 & 0 & 0 & 0 & 0 & 0 & 5 & 5 \\
\hline Total & 10 & 10 & 5 & 5 & 5 & 10 & 10 & 5 & 5 & 5 & 10 & 10 & 95 \\
\hline \multicolumn{14}{|l|}{ Molecular-Copro Ring Trial test } \\
\hline rt-PCR ${ }^{1}$ (Tsol 9) [30,31] & 0 & 5 & 0 & 0 & 0 & 0 & 0 & 0 & 0 & 0 & 0 & 0 & 5 \\
\hline rt-PCR (HDP2) [32] & 0 & 5 & 0 & 0 & 0 & 0 & 0 & 0 & 0 & 0 & 0 & 0 & 5 \\
\hline c-PCR ${ }^{2}$ (Tsol 9) $[30,31]$ & 0 & 5 & 0 & 0 & 0 & 0 & 0 & 0 & 0 & 0 & 0 & 0 & 5 \\
\hline c-PCR (HDP2) [32] & 0 & 5 & 0 & 0 & 0 & 0 & 0 & 0 & 0 & 0 & 0 & 0 & 5 \\
\hline PCR [33] & 5 & 0 & 0 & 0 & 0 & 0 & 0 & 0 & 0 & 0 & 0 & 0 & 5 \\
\hline PCR [34] & 5 & 0 & 0 & 0 & 0 & 0 & 0 & 0 & 0 & 0 & 0 & 0 & 5 \\
\hline PCR [35] & 0 & 0 & 0 & 0 & 0 & 0 & 5 & 0 & 0 & 0 & 0 & 0 & 5 \\
\hline c-PCR (ITS) & 0 & 0 & 0 & 0 & 0 & 0 & 0 & 0 & 5 & 0 & 0 & 0 & 5 \\
\hline PCR [30] & 0 & 0 & 5 & 0 & 0 & 0 & 0 & 0 & 0 & 0 & 0 & 0 & 5 \\
\hline PCR-RFLP ${ }^{3}$ [36] & 0 & 0 & 5 & 0 & 0 & 0 & 0 & 0 & 0 & 0 & 0 & 0 & 5 \\
\hline Semi-nested PCR + RFLP [37] & 0 & 0 & 0 & 5 & 0 & 0 & 0 & 0 & 0 & 0 & 0 & 0 & 5 \\
\hline Sanger sequencing & 5 & 0 & 0 & 0 & 0 & 0 & 0 & 0 & 0 & 0 & 0 & 0 & 5 \\
\hline Total & 15 & 20 & 10 & 5 & 0 & 0 & 5 & 0 & 5 & 0 & 0 & 0 & 60 \\
\hline
\end{tabular}

${ }^{1}$ real time-PCR; ${ }^{2}$ conventional-PCR; ${ }^{3}$ PCR-restriction fragment length polymorphism.

Table 11. Diagnostic accuracy by test of the microscopic copro-Ring Trial.

\begin{tabular}{|c|c|c|c|c|c|c|c|c|c|c|}
\hline Test & $\mathrm{TN}^{1}$ & $\mathrm{FP}^{2}$ & $\mathrm{FN}^{3}$ & $\mathrm{TP}^{4}$ & $\begin{array}{l}\text { Total } \\
\text { Samples }\end{array}$ & $\begin{array}{l}\text { Sensitivity } \\
(\%)\end{array}$ & $\begin{array}{l}\text { Specificity } \\
\quad(\%)\end{array}$ & $\begin{array}{c}\text { Correctly } \\
\text { Classified } \\
\text { (Reliability) }\end{array}$ & $\begin{array}{c}\text { ROC } \\
\text { Area } \\
\text { (Accuracy) }\end{array}$ & $95 \%$ IC \\
\hline $\begin{array}{l}\text { Saline and iodine wet mount } \\
\text { preparations of fecal smears } \\
\text { for direct examination }\end{array}$ & 14 & 0 & 0 & 21 & 35 & 100 & 100 & 100 & 1 & $(0.99-1.0)$ \\
\hline $\begin{array}{l}\text { Microscopic examination } \\
\text { after concentration (whether } \\
\text { by sedimentation or } \\
\text { flotation) }\end{array}$ & 21 & 1 & 1 & 32 & 55 & 96.97 & 95.45 & 96.36 & 0.962 & $(0.87-0.99)$ \\
\hline $\begin{array}{l}\text { Kato-Katz followed by } \\
\text { Ziehl-Neelsen staining [29] }\end{array}$ & 2 & 0 & 0 & 3 & 5 & 100 & 100 & 100 & 1 & $(0.48-1.0)$ \\
\hline
\end{tabular}

${ }^{1}$ True negative; ${ }^{2}$ false positive; ${ }^{3}$ false negative; ${ }^{4}$ true positive.

The six laboratories participating in the molecular-coprological RT (Table 1) used a number of different PCR protocols (Table 10). The performance of the assays used is shown in Table 12. No false positives were recorded by any of the participating laboratories for the negative control sample and also for the E. granulosus DNA-spiked sample (Table S2). Only 1 laboratory (17\%) (Lab L) correctly identified all 3 positive samples and only a single laboratory (Lab S) registered false negatives for all the 3 true positive samples. Overall, 5 laboratories (83\%) (B, C, D, E, L) detected T. solium spiked DNA-sample whereas only 1 laboratory (17\%) (Lab L) correctly identified the T. saginata DNA-spiked sample. The sample spiked with $T$. solium proglottids was confirmed by 3 laboratories $(50 \%)(C, D, L)$. 
Table 12. Diagnostic performance by test of the molecular copro-Ring Trial.

\begin{tabular}{|c|c|c|c|c|c|c|c|c|}
\hline Molecular-Test & $\mathrm{TN}^{1}$ & $\mathrm{FP}^{2}$ & $\mathrm{FN}^{3}$ & $\mathrm{TP}^{4}$ & $\begin{array}{c}\text { Total } \\
\text { Samples }\end{array}$ & $\begin{array}{c}\text { Sensitivity } \\
(\%)\end{array}$ & $\begin{array}{l}\text { Specificity } \\
(\%)\end{array}$ & $\begin{array}{l}\text { Correctly Classified } \\
\text { (Reliability, \%) }\end{array}$ \\
\hline rt-PCR ${ }^{5}$ (Tsol 9) $[30,31]$ & 2 & 0 & 1 & 2 & 5 & 66.6 & 100 & 80 \\
\hline rt-PCR (HDP2) [32] & 2 & 0 & 2 & 1 & 5 & 33.3 & 100 & 60 \\
\hline c- PCR ${ }^{6}$ (Tsol 9) $[30,31]$ & 2 & 0 & 2 & 1 & 5 & 33.3 & 100 & 60 \\
\hline c- PCR (HDP2) [32] & 2 & 0 & 2 & 1 & 5 & 33.3 & 100 & 60 \\
\hline PCR [33] & 2 & 0 & 3 & 0 & 5 & - & 100 & 40 \\
\hline PCR 34] & 2 & 0 & 2 & 1 & 5 & 33.3 & 100 & 60 \\
\hline PCR [35] & 2 & 0 & 0 & 3 & 5 & 100 & 100 & 100 \\
\hline c-PCR (ITS) & 2 & 0 & 3 & 0 & 5 & - & 100 & 40 \\
\hline PCR [30] & 2 & 0 & 2 & 1 & 5 & 33.3 & 100 & 60 \\
\hline PCR-RFLP ${ }^{7}$ [36] & 2 & 0 & 2 & 1 & 5 & 33.3 & 100 & 60 \\
\hline Semi-nested PCR + RFLP [37] & 2 & 0 & 2 & 1 & 5 & 33.3 & 100 & 60 \\
\hline Sanger sequencing & 2 & 0 & 2 & 1 & 5 & 33.3 & 100 & 60 \\
\hline
\end{tabular}

${ }^{1}$ True negative; ${ }^{2}$ false positive; ${ }^{3}$ false negative; ${ }^{4}$ true positive; ${ }^{5}$ real time PCR; ${ }^{6}$ conventional PCR; ${ }^{7}$ PCR-restriction fragment length polymorphism.

\section{Discussion}

Ring trials using well characterized clinical samples are essential to assess the performance of diagnostic tests, to harmonize and standardize their use, and importantly to ascertain their reproducibility and relevance to clinical work. The main limitations of this collaborative study include the low number of well characterized clinical samples; incomplete clinical information for some of the samples; and the lack of samples from patients with similar clinical presentations (such as brain tumours, metastases, or epileptic seizures) to establish specificity in clinical settings. The acquisition of clinical samples specific to regions where Taenia spp. infections have a rare occurrence as for instance in Europe is problematic [12,13,38]. However, despite these limitations, it has been possible to amass data on diagnostic tools used for the detection of Taenia spp. infections in humans as well as information on their performance in the hands of European laboratories.

Laboratories participating in the first serological RT used 11 tests. Analysing all the results together, sensitivity was low $(67.6 \%, 95 \%$ CI $59.1-75.4)$ and only $77.45 \%$ of the samples were correctly classified (i.e., correctely identified as positive samples). Four out of 11 tests were based on WB, five on ELISA and two were assays that are no longer commercially available (Table 4). In spite of studies showing the low diagnostic performance of these ELISAs [17,39-41], some European laboratories are still using these tests for NCC diagnosis [5] since these assays are easy to use particularly due to the convenience of automated reading. In recent years, several ELISA-based tests were developed and commercialized for the diagnosis of NCC. However, independent information regarding the specificity and sensitivity of these tests is often unavailable. The diagnostic specifications of these kits are reported in the kit insert, but usually they are not informative enough to establish the clinical performance of the assay. Since most of the laboratories have no or limited expertise on diagnosing NCC, the selection of a commercial kit for routine diagnosis can be difficult due to the lack of independent validation data, the lack of well-defined serum samples to validate the test and limited requests from clinicians. Additionally, shelf lifetime and cost are increasingly important, and diagnostic performance is difficult to establish. In the first serological RT, the LLGP-EITB [26] showed the best performance in terms of sensitivity $(91.87 \%)$ and accuracy $(0.958,95 \%$ CI $0.85-0.99)$, followed by Cypress Diagnostics ${ }^{\circledR}$ (Hulshoult, Belgium) (Table 5). The lowest accuracy was recorded for the CIE, which did not detect any positives and only correctly classified the negative samples, followed by Novagnost ${ }^{\circledR}(0.6875$ accuracy, $95 \%$ CI $0.35-0.90$; Table 5$)$. The in-house IFA, an outdated test which is no longer commercially available, showed similar or higher accuracy than some of the other tests such as Bioactiva Diagnostica ${ }^{\circledR}$ (Bad Homburg, Germany), GTS-T24H [25], Novagnost ${ }^{\circledR}$ (NovaTec Immunodiagnostica GmbH, Dietzenbach, Germany), NovaLisa ${ }^{\circledR}$ (Novatec Immundiagnostica GmbH, Dietzenbach, Germany), r-EITB [27], r-T24H EITB [25], and LDBIO Diagnostics ${ }^{\circledR}$ (Lyon, France ) (Table 5). However, the evaluation of a test used uniquely by one laboratory (i.e., Bioactiva Diagnostica ${ }^{\circledR}$ (Bad Homburg, Germany), 
Cypress Diagnostics ${ }^{\circledR}$ (Hulshoult, Belgium), GTS-T24H [25], Novagnost ${ }^{\circledR}$, NovaTec Immunodiagnostica GmbH, Dietzenbach, Germany), r-EITB [27], r-T24H EITB [25], IFA, and CIE; Table 5) is negatively influenced by sample size, which may have led to the wide $95 \%$ CI observed in this study, when the accuracy was determined. In a previous study using a larger panel of samples [40], a sensitivity of $64.3 \%$ was recorded for Cypress Diagnostics ${ }^{\circledR}$ (Hulshoult, Belgium), which is lower than the sensitivity $(87.5 \%)$ detected in the present study, but similar to that of LLGP-EITB [26] (Table 5). In a recent study [41], the diagnostic value of r-T24H EITB [25] was considered similar to that of LLGP-EITB [26], whereas, in the present study, both tests performed differently (Table 5 ). This disparity may be related to the individual laboratories, since test accuracy may be influenced by laboratory performance. Additionally, the observed differences may be related to location, number and nature of cysts as well as to low IgG levels. The highest accuracy for the LLGP-EITB [26] test was achieved by laboratory C followed by laboratories B and L (Table 6). However, despite differences in accuracy, the $\mathrm{K}$ values show a good level of agreement among laboratories performing this test (Table 7). In the first serological RT, LLGP-EITB [26] was significantly less associated with false-negatives than LDBIO Diagnostics ${ }^{\circledR}$ (Lyon, France) OR 0.2; 95\% CI 0.04-1.02; $p<0.05$ ) and NovaLisa ${ }^{\circledR}$ (Novatec Immundiagnostica GmbH, Dietzenbach, Germany) (OR 6.6; 95\% CI 1.128-38.60; $p<0.036$ ).

Most negative samples were correctly classified (i.e., correctely identified as negative samples). The influence of cyst localization in NCC patients was restricted to positive samples. Independent of the test and the laboratory, the percentage of false negative results was higher for samples from individuals with intra-parenchymal cysts $(39.2 \%)$ than those from patients with extra-parenchymal lesions (11.8\%) (Table 8$)$. In addition, considering both laboratory or laboratory and test, a significantly higher OR $(p<0.004)$ was recorded for patients with intra-parenchymal lesions as opposed to those with extra-parenchymal ones (Table 9). This is in accordance with previous reports that described a higher antibody concentration in serum samples from extra-parenchymal NCC patients and, consequently, they are more easily detectable by a broad range of tests [17,23,42-44].

Only three laboratories tested the panel of sera by an antigen detection assay (Table 4). The known lower diagnostic sensitivity of this assay in comparison to those based on antibody detection $[17,45]$ could be the reason for excluding this assay from the routine practice of many European laboratories. Sensitivities of $62.5 \%$ and $43.7 \%$ and accuracies of 0.81 and 0.66 were achieved for the commercial and in-house antigen detection assay, respectively (Table 5). In contrast to antibody detecting tests, antigen detecting assays only show positive results in the presence of viable cysticerci, which may explain their lower performance in this study. Antigen detection tests are of interest for the clinician to triage patients for neuroimaging in low-resource settings, support therapy options in association with the neuroimaging results and assess the outcome of anthelmintic treatment [17]. The inter-rater agreement for the circulating antigen test performed by two laboratories showed a $\mathrm{K}$ value of 0.66 , which was considered substantial (Table 7).

To confirm the results of the first serological RT, a second serological RT was organized. To this end, the three assays that were most frequently used during the first RT were selected, consequently increasing sample size per test. The total sensitivity was again low (52.8\%). The LLGP-EITB [26] once more showed the highest performance (Table 5). These results support previous data showing higher diagnostic performance of WB in comparison to other assays $[17,45]$. Since laboratory performances are known to influence test results, laboratories should carefully check all the assay steps to ensure high standards are maintained. The participation of laboratories in consecutive RTs may positively affect laboratory performance. Furthermore, every new diagnostic assay should go through an external quality assessment service.

The results of the copro RT suggest that generally the European laboratories appear to be better equipped for traditional microscopy than for molecular diagnosis of taeniosis, since only half of the laboratories participating in the microscopy testing also performed the molecular-coprological RT (Table 8). Using traditional coprological methods, 91.7\% 
of laboratories correctly identified all the samples. This preference for the coprological tests, observed in a previous study [5], can be explained by the low cost associated with microscopic coprological diagnosis that is routinely performed for most intestinal parasites, including protozoa and helminths. The laboratory performances of the copro RT were very high with an accuracy close to 1 (Table 11). Conversely, the molecular methods did not reach the expected level of performance, showing a sensitivity lower than 75\% (Table 11).

Two DNA-spiked fecal samples were used in this copro RT due to difficulties in obtaining material from clinical patients. Despite this, spiked DNA of T. solium was retrieved by $83 \%$ of participants. In contrast only one laboratory was able to detect T. saginata spiked DNA. This may be a factor of both operator performance and sensitivity of the PCR assay used and is evident in the fact that only half of the participating laboratories were able to detect $T$. solium DNA in a sample from a confirmed patient with egg-laden proglottids (Table S2). Further to this, results recorded by the six participating laboratories were obtained using a mix of assays by which the positive RT samples were positive using a given PCR assay and negative with another (Table S2). This illustrates the need for the acquisition of clinical samples from confirmed taeniosis patients to be used in the standardization and harmonization of PCR protocols. This should be carried out within an external quality assessment service.

\section{Conclusions}

Based on the comparison of tests currently in use for the serological diagnosis of NCC by European laboratories, the use of a WB-based method appears advantageous. Laboratories should select the serological diagnostic kits based on their diagnostic performance rather than on cost and ease of use. For clinicians, our results confirm a high specificity, but relatively low sensitivity for $T$. solium antibodies or antigens.

To further evaluate the sensitivity and specificity of the assays tested in this study, additional biological samples positive for Taenia spp. from patients with confirmed taeniosis/cysticercosis originating from endemic regions should be tested.

The European laboratories performed well with regard to carrying out stool examination based on microscopy for detecting Taenia spp. infections. Copro-molecular techniques need standardization, validation and harmonization, and external quality assessment services. This is extremely important as molecular tools are relevant for Taenia species, allowing the identification of T. solium carriers, which may point the way to symptomatic or asymptomatic NCC cases and has clear therapeutic consequences.

Supplementary Materials: The following are available online at https:/ / www.mdpi.com/article/ 10.3390/microorganisms9061173/s1, Supplementary Table S1: Breakdown of the microscopiccoprological Ring Trial results; Supplementary Table S2: Breakdown of the molecular-coprological Ring Trial results.

Author Contributions: All authors have made substantial contributions to the conception of the work and interpretation of data and has approved the submitted version. M.Á.G.-M., A.L., B.B., P.D., T.K., J.B., S.G., V.S., E.P. and A.S.W. conceived and planned the work. C.A., H.A., E.B., S.B., C.D.C., Z.D., M.J-P., P.M., E.P., M.F., M.J.P., I.R.-O., B.S., C.R., P.D., T.K., A.L. and M.A. tested the panel of samples. M.A.G.M. and B.B., extracted the data. M.Á.G.-M., P.P., A.L. and B.B. analysed the data. M.Á.G.-M., B.B. and E.P. drafted the manuscript and prepared the tables. All authors contributed in revising and editing the manuscript. All authors have read and agreed to the published version of the manuscript.

Funding: The authors would like to acknowledge financial support for the delivery of samples provided by the Centre for Global Health, Institute of Health and Society, University of Oslo, and in particular, Mrs. Ekaterina Bogatyreva and Mrs. Ingeborg K Haavardsson. This work was a collaboration within the framework of CYSTINET, the European network on taeniosis/cysticercosis, COST ACTION TD1302.

Institutional Review Board Statement: The study was conducted according to the guidelines of the Declaration of Helsinki Ethical. Review and approval were waived for this study, due to the fact 
that samples had been collected by participants following informed consent from people for their use in clinical studies peformed along years in several laboratories around the world. Anonemized samples were stored under the required conditions $\left(-20^{\circ} \mathrm{C}\right.$ or $\left.-80^{\circ} \mathrm{C}\right)$ in respective laboratories for a number of years. Participant laboratories shared their samples for this study.

Informed Consent Statement: Informed consent was obtained from all subjects involved in the study.

Data Availability Statement: Data are available after request.

Acknowledgments: This work is dedicated to Teresa Gárate who passed away at the end of these collaborative studies. She was a highly respected Parasitologist, devoting her life to understanding aspects of host-parasite interactions. She provided her expertise with intelligence and generosity. We will always remember her as a wonderful individual and true scientist. We are very grateful to Evans Kabemba Mwape, Istvan Kuscera, and Lucy Robertson, who provided human samples.

Conflicts of Interest: The authors declare no conflict of interest.

\section{References}

1. Del Brutto, O.H. Neurocysticercosis. Semin. Neurol. 2005, 25, 243-251. [CrossRef]

2. Zammarchi, L.; Strohmeyer, M.; Bartalesi, F.; Bruno, E.; Muñoz, J.; Buonfrate, D.; Nicoletti, A.; García, H.H.; Pozio, E.; Bartoloni, A. COHEMI Project Study Group. Epidemiology and management of cysticercosis and Taenia solium taeniasis in Europe, systematic review 1990-2011. PLoS ONE 2013. [CrossRef]

3. Torgerson, P.R.; Devleesschauwer, B.; Praet, N.; Speybroeck, N.; Willingham, A.L.; Kasuga, F.; Rokni, M.B.; Zhou, X.N.; Fèvre, E.M.; Sripa, B.; et al. World Health Organization estimates of the global and regional disease burden of 11 foodborne parasitic diseases, 2010: A data synthesis. PLoS Med. 2015. [CrossRef]

4. Dorny, P.; Praet, N. Taenia saginata in Europe. Vet. Parasitol. 2007, 149, 22-24. [CrossRef]

5. Gómez-Morales, M.A.; Gárate, T.; Blocher, J.; Devleesschauwer, B.; Schmidt, G.S.A.; Perteguer, M.J.; Ludovisi, A.; Pozio, E.; Dorny, P.; Gabriël, S.; et al. Present status of laboratory diagnosis of human taeniosis/cysticercosis in Europe. Eur. J. Clin. Microbiol. Infect. Dis. 2017, 36, 2029-2040. [CrossRef]

6. Murrell, K.D. Epidemiology of taeniosis and cysticercosis. In WHO/FAO/OIE Guidelines for the Surveillance, Prevention and Control of Taeniosis/Cysticercosis; Murrell, K.D., Ed.; OIE (World Organisation for Animal Health); WHO (World Health Organization); FAO (Food and Agriculture Organization): Paris, France, 2005; pp. 27-43. Available online: https://www.oie.int/doc/ged/d11245.pdf (accessed on 30 March 2021).

7. Winkler, A.S. Neurocysticercosis in sub-Saharan Africa: A review of prevalence, clinical characteristics, diagnosis, and management. Pathog. Glob. Health 2012, 106, 261-274. [CrossRef]

8. Winkler, A.S.; Richter, H.; Landscape analysis: Management of Neurocysticercosis with an Emphasis on Low- and Middle-Income Countries. World Health Organization. 2015. Available online: https://apps.who.int/iris/bitstream/handle/10665/152896 /WHO_HTM_NTD_NZD_2015.05_eng.pdf?sequence=1 (accessed on 30 March 2021).

9. Devleesschauwer, B.; Allepuz, A.; Dermauw, V.; Johansen, M.V.; Laranjo-González, M.; Smit, G.S.; Sotiraki, S.; Trevisan, C.; Wardrop, N.A.; Dorny, P.; et al. Taenia solium in Europe: Still endemic? Acta Trop. 2017, 165, 96-99. [CrossRef]

10. Fabiani, S.; Bruschi, F. Neurocysticercosis in Europe: Still a public health concern not only for imported cases. Acta Trop. 2013, 128, 18-26. [CrossRef] [PubMed]

11. Van de Pol, L.A.; van Doeveren, T.E.; van der Kuip, M.; Wolf, N.I.; Vermeulen, R.J. Pediatric neurocysticercosis: Three cases presented in the Netherlands with divergent clinical presentations. Neuropediatrics 2015, 46, 130-133. [CrossRef] [PubMed]

12. Laranjo-González, M.; Devleesschauwer, B.; Trevisan, C.; Allepuz, A.; Sotiraki, S.; Abraham, A.; Afonso, M.B.; Blocher, J.; Cardoso, L.; Correia da Costa, J.M.; et al. Epidemiology of taeniosis/cysticercosis in Europe, a systematic review: Western Europe. Parasit. Vectors. 2017, 10, 349. [CrossRef] [PubMed]

13. Trevisan, C. ; Sotiraki; S. ; Laranjo-González, M.; Dermauw, V.; Wang, Z.; Kärssin, A.; Cvetkovikj, A.; Winkler, A.S.; Abraham, A.; Bobić, B.; et al. Epidemiology of taeniosis/cysticercosis in Europe, a systematic review: Eastern Europe. Parasit Vectors. 2018, 11, 569. [CrossRef]

14. Gabriël, S.; Johansen, M.V.; Pozio, E.; Smit, G.S.; Devleesschauwer, B.; Allepuz, A.; Papadopoulos, E.; van der Giessen, J.; Dorny, P. Human migration and pig/pork import in the European Union: What are the implications for Taenia solium infections? Vet. Parasitol. 2015, 213, 38-45. [CrossRef]

15. Food and Agriculture Organization of the United Nations (FAO)/World Health Organization (WHO). Multicriteria-Based Ranking for Risk Management of Food-Borne Parasites. Microbiological Risk Assessment, Series No. 23; FAO/WHO: Rome, Italy, 2014; Available online: http:/ / www.fao.org/publications/card/en/c/ee07c6ae-b86c-4d5f-915c-94c93ded7d9e/ (accessed on 30 March 2021).

16. Bouwknegt, M.; Devleesschauwer, B.; Graham, H.; Robertson, L.; Van der Giessen, J.; the Euro-FBP Workshop Participants. Prioritisation of food-borne parasites in Europe. Euro Surveill. 2018, 23, 17-00161. [CrossRef]

17. Del Brutto, O.H.; Nash, T.E.; White, A.C. Jr; Rajshekhar, V.; Wilkins, P.P. , Singh, G.; Vasquez; C.M.; Salgado, P.; Gilman; R.H.; Garcia, H.H. Revised diagnostic criteria for neurocysticercosis. J. Neurol. Sci. 2017, 372, 202-210. [CrossRef] [PubMed]

18. Winkler, A.S.; Willingham, A.L.; Sikasunge, C.S.; Schmutzhard, E. Epilepsy and neurocysticercosis in sub-Saharan Africa. Wien Klin. Wochenschr. 2009, 121, 3-12. [CrossRef] [PubMed] 
19. Clinton White, A., Jr.; Coyle, C.M.; Rajshekhar, V.; Singh, G.; Hauser, W.A.; Mohanty, A.; Garcia, H.H.; Nash, T.E. Guidelines by the Infectious Diseases Society of America (IDSA) and the American Society of Tropical Medicine and Hygiene (ASTMH). Clin. Infect. Dis. 2018, 66, 49-75. [CrossRef]

20. CYSTINET, the European Network on Taeniosis/Cysticercosis. Available online: https:/ / cystinet.org (accessed on 11 March 2021$).$

21. Tamburrini, A.; Gómez-Morales, M.A.; Pozio, E. Development of an immunoenzyme test for the diagnosis of human cysticercosis using a heterologous antigen. Parassitologia 1995, 37, 195-198. [PubMed]

22. Mwape, K.E.; Phiri, I.K.; Praet, N.; Muma, J.B.; Zulu, G.; Van den Bossche, P. , de Deken, R.; Speybroeck, N.; Dorny, P.; Gabriël, S. Taenia solium infections in a rural area of Eastern Zambia-a community-based study. PLoS Negl. Trop Dis. 2012, 6, e1594. [CrossRef]

23. Rodriguez, S.; Dorny, P.; Tsang, V.C.; Pretell, E.J.; Brandt, J.; Lescano, A.G.; Gonzalez, A.E.; Gilman, R.H.; Garcia, H.H. Cysticercosis Working Group in Peru. Detection of Taenia solium antigens and anti-T. solium antibodies in paired serum and cerebrospinal fluid samples from patients with intraparenchymal or extraparenchymal neurocysticercosis. J. Infect. Dis. 2009, 199, $1345-1352$. [CrossRef]

24. Landis, J.; Koch, G. The measurement of observer agreement for categorical data. Biometrics 1977, 33, 159-174. [CrossRef]

25. Hernández-González, A.; Noh, J.; Perteguer, M.J.; Gárate, T.; Handali, S. Comparison of T24H-his, GST-T24H and GST-Ts8B2 recombinant antigens in western blot, ELISA and multiplex bead-based assay for diagnosis of neurocysticercosis. Parasit. Vectors 2017, 10, 237. [CrossRef]

26. Tsang, V.C.; Brand, J.A.; Boyer, A.E. An enzyme-linked immunoelectrotransfer blot assay and glycoprotein antigens for diagnosing human cysticercosis (Taenia solium). J. Infect. Dis. 1989, 159, 50-59. [CrossRef] [PubMed]

27. Noh, J.; Rodriguez, S.; Lee, Y.M.; Handali, S.; Gonzalez, A.E.; Gilman, R.H.; Tsang, V.C.; Garcia, H.H.; Wilkins, P.P. Recombinant protein- and synthetic peptide-based immunoblot test for diagnosis of neurocysticercosis. J. Clin. Microbiol. 2014, 52, 1429-1434. [CrossRef] [PubMed]

28. Dorny, P.; Phiri, I.K.; Vercruysse, J.; Gabriel, S.; Willingham, A.L.; Brandt, J.; Victor, B.; Speybroeck, N.; Berkvens, D.A. Bayesian approach for estimating values for prevalence and diagnostic test characteristics of porcine cysticercosis. Int. J. Parasitol. 2004, 34, 569-576. [CrossRef] [PubMed]

29. Jimenez, J.A.; Rodriguez, S.; Moyano, L.M.; Castillo, Y.; García, H.H. for the Cysticercosis Working Group in Peru. Differentiating Taenia eggs found in human stools-Does Ziehl Neelsen staining help? Trop. Med. Int. Health 2010, 15, 1077-1081. [CrossRef] [PubMed]

30. Almeida, C.R.; Ojopi, E.P.; Nunes, C.M.; Machado, L.R.; Takayanagui; O. M.; Livramento, J.A.; Abraham, R.; Gattaz, W.F.; Vaz, A.J.; Dias-Neto, E. Taenia solium DNA is present in the cerebrospinal fluid of neurocysticercosis patients and can be used for diagnosis. Eur. Arch. Psychiatry Clin. Neurosci. 2006, 256, 307-310. [CrossRef] [PubMed]

31. Yera, H.; Dupont, D.; Houze, S.; Ben M'rad, M.; Pilleux, F.; Sulahian, A.; Gatey, C.; Gay Andrieu, F.; Dupouy-Camet, J. Confirmation and follow-up of neurocysticercosis by real-time PCR in cerebrospinal fluid samples of patients living in France. J. Clin. Microbiol. 2011, 49, 4338-4340. [CrossRef]

32. Hernández, M.; Gonzalez, L.M.; Fleury, A.; Saenz, B.; Parkhouse, R.M.; Harrison, L.J.; Garate, T.; Sciutto, E. Neurocysticercosis: Detection of Taenia solium DNA in human cerebrospinal fluid using a semi-nested PCR based on HDP2. Ann. Trop Med. Parasitol. 2008, 102, 317-323. [CrossRef] [PubMed]

33. Trachsel, D.; Deplazes, P.; Mathis, A. Identification of taeniid eggs in the faeces from carnivores based on multiplex PCR using targets in mitochondrial DNA. Parasitology 2007, 134, 911-920. [CrossRef]

34. Roelfsema, J.H.; Nozari, N.; Pinelli, E.; Kortbeek, L.M. Novel PCRs for differential diagnosis of cestodes. Exp. Parasitol. 2016, 161, 20-26. [CrossRef]

35. Yamasaki, H.; Allan, J.C.; Sato, M.O.; Nakao, M.; Sako, Y.; Nakaya, K.; Qiu, D.; Mamuti, W.; Craig, P.S.; Ito, A. DNA differential diagnosis of taeniasis and cysticercosis by multiplex PCR. J. Clin. Microbiol. 2004, 42, 548-553. [CrossRef] [PubMed]

36. Rodriguez-Hidalgo, R.; Geysen, D.; Benítez-Ortiz, W.; Geerts, S.; Brandt, J. Comparison of conventional techniques to differentiate between Taenia solium and Taenia saginata and an improved polymerase chain reaction-restriction fragment length polymorphism assay using a mitochondrial 12S rDNA fragment. J. Parasitol. 2002, 88, 1007-1011. [CrossRef]

37. Geysen, D.; Kanobana, K.; Victor, B.; Rodriguez-Hidalgo, R.; De Borchgrave, J.; Brandt, J.; Dorny, P. Validation of meat inspection results for Taenia saginata cysticercosis by PCR-restriction fragment length polymorphism. J. Food Prot. 2007, 70, 236-240. [CrossRef] [PubMed]

38. Abraham, A.; Schmidt, V.; Kaminski, M.; Stelzle, D.; De Meijere, R.; Bustos, J.; Sahu, P.S.; Garcia, H.H.; Bobić, B.; Cretu, C.; Chiodini, P.; et al. Epidemiology and surveillance of human (neuro)cysticercosis in Europe: Is enhanced surveillance required? Trop Med. Int. Health 2020, 25, 566-578. [CrossRef] [PubMed]

39. Proaño-Narvaez, J.V.; Meza-Lucas, A.; Mata-Ruiz, O.; García-Jerónimo, R.C.; Correa, D. Laboratory diagnosis of human neurocysticercosis: Double-blind comparison of enzyme-linked immunosorbent assay and electroimmunotransfer blot assay. $J$. Clin. Microbiol. 2002, 40, 2115-2118. [CrossRef] [PubMed]

40. Carod, J.F.; Randrianarison, M.; Razafimahefa, J.; Ramahefarisoa, R.M.; Rakotondrazaka, M.; Debruyne, M.; Dautigny, M.; Cazal, P.; Andriantseheno, M.L.; Charles, E.R. Evaluation of the performance of 5 commercialized enzyme immunoassays for the detection of Taenia solium antibodies and for the diagnosis of neurocysticercosis. Diagn. Microbiol. Infect. Dis. 2012, 72, 85-89. [CrossRef] 
41. Dermauw, V.; Carabin, H.; Cissé, A.; Millogo, A.; Tarnagda, Z.; Ganaba, R.; Noh, J.; Handali, S.; Breen, K.; Richter, V.; et al. Evaluating the Recombinant T24H Enzyme-Linked Immunoelectrotransfer Blot Assay for the Diagnosis of Neurocysticercosis in a Panel of Samples from a Large Community-Based Randomized Control Trial in 60 Villages in Burkina Faso. Am. J. Trop. Med. Hyg. 2018, 98, 565-569. [CrossRef]

42. Zini, D.; Farrell, V.J.; Wadee, A.A. The relationship of antibody levels to the clinical spectrum of human neurocysticercosis. J. Neurol. Neurosurg. Psychiatry 1990, 53, 656-661. [CrossRef]

43. Michelet, L.; Fleury, A.; Sciutto, E.; Kendjo, E.; Fragoso, G.; Paris, L.; Bouteille, B. Human neurocysticercosis: Comparison of different diagnostic tests using cerebrospinal fluid. J. Clin. Microbiol. 2011, 49, 195-200. [CrossRef]

44. Garcia, H.H.; Nash, T.E.; Del Brutto, O. Clinical symptoms, diagnosis, and treatment of neurocysticercosis. Lancet Neurol. 2014, 13, 1202-1215. [CrossRef]

45. Garcia, H.H., Castillo; Gonzales, I., Bustos; Saavedra, H.; Jacob, L.; Del Brutto, O.H.; Wilkins, P.P.; Gonzalez, A.E.; Gilman, R.H. Cysticercosis Working Group in Peru. Low sensitivity and frequent cross-reactions in commercially available antibody detection ELISA assays for Taenia solium cysticercosis. Trop. Med. Int. Health 2018, 23, 101-105. [CrossRef] [PubMed] 